\section{New drugs cost US\$2.6 billion to develop}

The cost of drug development, including the price of failure and the opportunity cost, has more than doubled in the past decade, shows a Tufts study. The lowdown: A widely cited 2003 study estimated that new drugs cost on average around US $\$ 800$ million (in 2000 dollars) to develop when you include the price of failures and a 'time cost', the expected returns that investors forgo while a drug is in development (lournal of Health Economics 22, 151-185; 2003). Adjusting for inflation alone, that should have translated into a cost

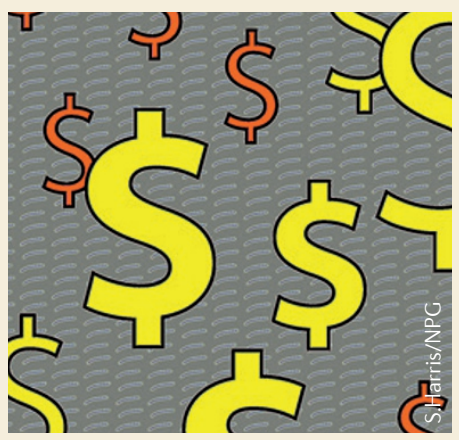
of just over $\$ 1$ billion in 2013. A new analysis by the same authors using similar methodology now instead puts the cost at $\$ 2.6$ billion in 2013 dollars, up by $145 \%$.

Joseph DiMasi, of the Tufts Center for the Study of Drug Development in Boston, Massachusetts, USA, and colleagues estimated drug development costs based on data provided by 10 drug companies for a randomly selected subset of 106 self-originated drugs that went into the clinic between 1995 and 2007. Of the included drugs, 87 were small-molecule chemical entities (three of which were synthetic peptides), 10 were monoclonal antibodies and 9 were recombinant proteins. The authors found that the average out-of-pocket contribution to the total cost was $\$ 1.4$ billion, and that the time cost was $\$ 1.2$ billion. As Nature Reviews Drug Discovery went to press, the failure rate estimates and the development- and review-timeline assumptions that were used to generate the cost data were not yet public.

DiMasi attributes the higher price tag largely to higher failure rates and to increasing out-of-pocket expenses, driven by factors such as increased clinical trial complexity, larger clinical trial sizes and the cost of collaborating with the medical sector.

The analysis also found that the average cost of post-approval studies was just over $\$ 300$ million.

DiMasi and his colleagues have submitted the article for peer review and publication.

\section{European regulators approve first PARP inhibitor}

But a US regulatory decision is still pending for AstraZeneca's olaparib.

The lowdown: In 2011 and 2012, a series of clinical trial failures threatened poly (ADP-ribose) polymerase (PARP) inhibitors - which modulate the repair of DNA damage - with extinction. Not only did Sanofi's iniparib fail in triple-negative breast cancer, but AstraZeneca's olaparib also disappointed in ovarian cancer. Subsequent studies showed that iniparib is not a functional PARP inhibitor and shed new light on patient selection, fuelling a come-back (Nature Rev. Drug Discov. 12, 725-727; 2013). Olaparib has now secured a full turnaround, receiving a vote of confidence from the European Medicines Agency (EMA) for its use as a monotherapy for the maintenance treatment of women with relapsed $B R C A$-positive ovarian cancer.

The EMA based their decision on a Phase II trial in 265 women with platinum-sensitive relapsed serous ovarian cancer. The median progression-free survival was 11.2 months with olaparib, versus 4.3 months on placebo (Lancet Oncol. 15, 852-861; 2014). At an interim analysis, overall survival did not differ between treatment groups. The most common adverse events associated with treatment were nausea, vomiting, fatigue and anaemia.

The US Food and Drug Administration was initially set to rule on the drug in October, but has postponed the decision date, probably until January 2015. The delay followed an independent advisory committee meeting in which panellists voted 11 to 2 against accelerated approval of the drug. Panellists debated the side-effect profile of the drug, the use of progression-free survival versus overall survival data, and the ethics of approving the drug while a Phase III confirmatory trial was still enrolling patients.

Olaparib is also in Phase III trials for breast cancer and in Phase II trials for non-small-cell lung cancer, prostate cancer and colorectal cancer.

Four other PARP inhibitor candidates are in hot pursuit. Merck and Tesaro's niraparib, Clovis' rucaparib, BioMarin's talazoparib and AbbVie's veliparib are all in Phase III trials for various oncology indications.

\section{Phase III setback for lead angiopoietin inhibitor}

Amgen and Takeda's trebananib peptibody missed its overall survival end point in a pivotal ovarian cancer trial, despite promising signals of progression-free survival.

The lowdown: Anti-angiogenesis drugs like Genentech/Roche's blockbuster bevacizumab — an antibody that inhibits vascular endothelial growth factor (VEGF) — offer efficacy in a range of oncology indications, but are limited by side effects including hypertension, thrombosis, emboli, bleeding, bowel perforation and central nervous system disorders. To side-step these adverse events, a few drug developers have targeted other drivers of angiogenesis, such as angiopoietin 1 (ANG1) and ANG2, which mediate vascular remodelling via the angiopoietin receptor TIE2. Amgen and Takeda had been leading the charge with their ANG1- and ANG2-binding peptibody trebananib which is made by fusing peptides to a crystallizable fragment Fc domain — but they have recently hit a snag.

In trebananib's first Phase III trial, the median overall survival was 19.3 months with the treatment, versus 18.3 months in the control arm, the drug developers reported in November. The firms had previously reported that the median progression-free survival was 7.2 months in the trebananib arm versus 5.4 months in the control arm. "We continue to explore the potential of trebananib's novel anti-tumor mechanism of action in other cancer settings," said Amgen's head of Research and Development, Sean Harper, in a statement.

Two other Phase III trials in ovarian cancer are ongoing. The firms are also testing the drug in Phase II trials in renal cell carcinoma, breast cancer and hepatocellular cancer.

At least three other ANG inhibitors are in clinical development. Roche's RG7221 is a bispecific antibody that binds both ANG2 and VEGFA. Roche initiated a Phase II trial of the bispecific therapy in patients with colorectal cancer in June. Medlmmune's ANG2-specific antibody MEDI3617 is in Phase I development. And Amgen's ANG1- and ANG2-targeting monoclonal antibody AMG 780 is in Phase I trials. Pfizer had previously made it as far as Phase II with its ANG2-specific CVX-060, but discontinued development of the antibody in 2013. 